http://doi.org/10.15359/ree.13-1.7

\title{
Multiplicando esfuerzos para fortalecer la familia desde la orientación
}

\author{
Eliud Calderón Monge ${ }^{l}$ \\ Subdirector de la División de Educación para el Trabajo, \\ Centro de Investigación y Docencia en Educación, Universidad Nacional \\ Heredia, Costa Rica \\ Ana Lucía Villalobos Cordero ${ }^{2}$ \\ Investigadora de la División de Educación para el Trabajo, \\ Centro de Investigación y Docencia en Educación, Universidad Nacional \\ Heredia, Costa Rica \\ Ruth Villanueva Barbarán ${ }^{3}$ \\ Investigadora y Académica de la División de Educación para el Trabajo, \\ Centro de Investigación y Docencia en Educación, Universidad Nacional \\ Heredia, Costa Rica
}

Recibido 13 de junio de 2008 • Aprobado 23 de marzo de 2009

Resumen. La propuesta rescata la sistematización de una experiencia de capacitación a 35 funcionarios y funcionarias docentes y administrativos provenientes de nueve escuelas de Educación General Básica, que se ubican en comunidades con características socioeconómicas desfavorables o de riesgo social. Esta capacitación fue desarrollada por un equipo de dos orientadoras y un orientador, cuyo propósito fundamental fue el facilitar en los y las asistentes, el desarrollo de habilidades que les permitan diseñar y desarrollar actividades en el ámbito de Escuela para padres y madres. Así, se ha modelado una propuesta de trabajo desde la orientación educativa, en busca de una mayor cobertura y eficacia

1 Máster en Orientación Educativa, Universidad de Wisconsin, USA. Subdirector de la División de Educación para el Trabajo, Centro de Investigación y Docencia en Educación, Universidad Nacional. Correo electrónico: ecalder@una.ac.cr

2 Licenciada en Ciencias de la Educación con énfasis en Orientación, Universidad de Costa Rica. Investigadora de la División de Educación para el Trabajo, Centro de Investigación y Docencia en Educación, Universidad Nacional. Correo electrónico: avil@una.ac.cr

3 Máster en Psicopedagogía, Universidad Estatal a Distancia. Licenciada en Ciencias de la Educación con énfasis en Orientación, Universidad de Costa Rica. Investigadora y Académica de la División de Educación para el Trabajo, Centro de Investigación y Docencia en Educación, Universidad Nacional.Correo electrónico: rvillanu@una.ac.cr 
de la labor orientadora con la familia desde las escuelas. Se pretende, con ello, promover relaciones familiares saludables que contribuyan al bienestar integral de la familia y, por ende, en el apropiado desenvolvimiento académico y socio afectivo de sus hijos e hijas estudiantes.

Palabras clave. Orientación, familia, padres, madres, escuela, educación no formal.

Abstract. The proposal includes the systematization of a training experience where 35 teaching and administrative officials participated. They came from 9 elementary schools located in marginal communities, developed by a team of two school counselors whose main purpose was to encourage among the participants the development of skills that will let them design and apply activities in the field of "parents' school".

Thus, an educational counseling proposal has been modeled in order to reach greater coverage and efficiency of the counseling work with families being done from within the school. Moreover, what's being sought is the promotion of healthy family relationships that contribute to the family's wholeness and well being and therefore to the appropriate academic and social development of their children.

Key words: Counseling, family, parents, school, non formal education.

\section{INTRODUCCIÓN}

Por la existencia de una gran variedad en su conformación, resulta difícil acuñar una definición comprensiva, universal y de aceptación general del concepto familia. En términos generales, se tiende a considerar la familia como la unidad social básica, sobre la que se fundamenta la sociedad. Según Pereira (2004, p.183), "la familia se compone de dos o más personas, relacionadas emocionalmente, las cuales viven en proximidad geográfica".

Los y las responsables de la construcción de una familia tienen el ineludible deber de proporcionar alimento, techo y abrigo a su descendencia, así como la natural obligación de brindarle guía en su formación como personas. A pesar de tratarse de necesidades que deben satisfacerse conjuntamente, existe mayor preocupación generalizada por suplir lo primero y con menor empeño lo segundo.

Otra inevitable responsabilidad de la familia es facilitar y apoyar a sus hijos e hijas en su vida escolar, hasta que alcancen sus metas ocupacionales o profesionales. Para ello, debe adoptar una postura asociativa y cooperativa con la institución escolar, traducida en acciones de apoyo mutuo y esfuerzos comunes para favorecer la formación integral de niños, niñas y jóvenes.

La familia es un ente social de presencia universal, con matices diferentes en algunas sociedades humanas, pero con un aspecto en común: en todas se da vida familiar. A pesar de las situaciones que se puedan presentar al interior de la familia, ésta sigue siendo la base de las relaciones sociales de los individuos, y en la que tiene origen la identidad personal (Zamudio, 2008).

En Costa Rica, el Proyecto Estado de la Nación (2006), ha venido informando que, a partir del año 2000 y por primera vez en la historia de la población mundial, hay más habitantes en la ciudad que en el campo. El país no ha escapado a esta tendencia. Por sus connotaciones sociológicas y psicológicas, esta realidad ha hecho que se incrementen las diversas formas de familia, tanto en número como en tipo.

Resulta innegable la trascendencia del papel que desempeña la familia en la conformación de la sociedad, y cómo, a la vez, ésta influye en la vida familiar. Castells (1997) afirma que "desde épocas remotas las sociedades humanas, han construido su organización social sobre la base de la estructura familiar" (p.55). De ahí la importancia de promover y fortalecer una apropiada vida en familia, si pretendemos apuntar a una sociedad con ciudadanos y ciudadanas que posean una mejor calidad de vida. 
Es evidente e indiscutible el hecho de que la familia es el primer contexto, así como el más importante, en el que se forman las personas, es en donde obtienen sus logros o enfrentan sus fracasos. Así pues, determinará la clase de ciudadanos y ciudadanas que van a ofrecer a la sociedad y, por ende, la clase de sociedad que va a generar. Esta afirmación no ignora ni subestima el aporte, también invaluable, que las instituciones educativas ofrecen a sus educandos. Padres, madres y docentes deben concebir a la educación como parte fundamental del proceso de la personalización que conduce al ser humano hacia su plenitud como persona.

\section{LA RELACIÓN DE LA FAMILIA Y LA ESCUELA}

La escuela y la familia son dos espacios en los que las niñas y los niños desarrollan su vida. La familia es el grupo donde inician su socialización, pero cuando ingresan al sistema formal de educación, la escuela también cobra una importancia significativa para ellas y ellos, en su desarrollo social.

La familia ha sufrido transformaciones en su dinámica interna. El ritmo con que se mueve la sociedad ha variado y muchos padres y madres, se ven inmersos en múltiples actividades que limitan el espacio para compartir en familia; aunado a esto, existe una constante necesidad de rendir, de manera eficiente, en las obligaciones laborales que desempeñan, situación que produce estrés, el que, muchas veces, es descargado en la misma familia.

El requerimiento de eficiencia laboral de padres y madres, junto a la necesidad de trabajar horas extras, o bien, tener más de un trabajo para cumplir con las obligaciones económicas, conlleva a una reducción del tiempo dedicado a la familia, por lo que, en algunos casos, los y las progenitores deben delegar su función de formadores y formadoras de seres humanos, a instituciones que se dedican al cuidado de niños y niñas o a la escuela. Esta tendencia disminuye el contacto entre los padres, las madres y sus hijos e hijas, quienes, generalmente, sólo se ven al final del día. Además, los niños y niñas pasan mucho tiempo en la escuela, con lo que se recarga sobre la institución educativa, también, la responsabilidad de atender necesidades en su formación integral.

El gran avance tecnológico de nuestra sociedad actual permite a los individuos accesar, constantemente, nueva e inagotable información. Esta realidad, en ocasiones, determina que las tareas educativas impliquen mayor grado de dificultad, necesidad de conocimientos y manejo tecnológico. En muchos casos, es difícil que los padres y las madres puedan asumir la tarea. Estas situaciones pueden generar un distanciamiento entre la familia y la escuela. Además, muchas veces, por la preocupación sobre el desenvolvimiento de sus hijos e hijas, pero por la falta de claridad de los roles que corresponden a cada cual, se genera una lucha de poderes entre estas instancias; las relaciones no son fluidas y, fácilmente, se crean conflictos, como consecuencia de una comunicación rígida o, bien, se opta por un divorcio entre ambas instituciones.

Los maestros y las maestras, así como los padres y las madres, deben buscar los medios para trabajar en forma conjunta, con el fin de atender las necesidades personales y académicas de los niños y las niñas. Para Pereira (2004):

Los padres y madres de familia tienen el derecho y el deber de conocer, tanto los objetivos y procedimientos de la escuela, como los programas educativos en que participan sus hijos. Los maestros deben estar enterados de las motivaciones, expectativas, valores, y características de las familias de sus alumnos. (p. 181) 
Un trabajo coordinado entre ambas instancias, traerá efectos substanciales y duraderos en la formación personal del niño y la niña, así como mayores posibilidades de un aprendizaje significativo y éxito escolar, lo que potencia la permanencia del estudiantado en el sistema educativo.

Bronfenbrenner (citado por Monereo y Solé, 1996) manifiesta que las posibilidades de desarrollo de los entornos en los que se desenvuelven los niños y las niñas se verá incrementado si los roles y las actividades en esos ambientes estimulan la aparición de ciertas condiciones, a saber: una confianza mutua, una orientación positiva, el consenso de metas y un creciente equilibrio de poderes entre los dos ámbitos, para el mejoramiento de la persona en desarrollo. Estas condiciones mencionadas, incrementarán el potencial educativo de la escuela y el hogar.

La confianza mutua implica que los y las docentes adopten una actitud comprensiva, aunque no estén completamente de acuerdo con el proceder de algunas familias. Rockwell (1995) afirma que un maestro sensible reconoce la importancia del sistema familiar y acepta las circunstancias que puedan darse en el hogar. Se debe partir del hecho de que si cierta familia no provee un contexto mejor para el estudiante no es porque no lo quiera, sino porque no sabe o no puede, a causa de múltiples factores. De esta manera, el maestro debe aceptar y valorar lo que la familia aporta. Este respeto mostrado por el docente podría dar como resultado la anuencia de la familia para recibir apoyo por parte de él o de otros profesionales, como por ejemplo: el orientador.

La orientación positiva se refiere a la disposición de destacar los aspectos valiosos que se posean, basarse en ellos para aprender a potenciarlos y practicarlos. Toda familia y todo docente posee fortalezas y debilidades; lo importante es concentrarse en las fortalezas. Muchas veces, debido a los cambios que ha tenido la estructura familiar, así como las múltiples ocupaciones de las madres y los padres, éstos se sienten inseguros de la labor de formadores que realizan. Al respecto, Rockwell (1995) afirma que las maestras y los maestros pueden empoderar a las madres y a los padres, para que se vean a sí mismos como gente capaz, ayudarles a ver sus fortalezas, sus opciones potenciales y sus elecciones.

Dar a conocer las finalidades y políticas escolares, así como indagar cuáles son las metas educativas de las familias, son acciones importantes para acrecentar el potencial formativo de ambas esferas. Partiendo de este conocimiento, se pueden dar intercambios entre el hogar y la escuela, para clarificar metas y prioridades de manera consensuada. Por supuesto que para ello se requiere de una apropiada comunicación entre las partes.

Generalmente, las familias que mantienen mayor acercamiento son aquellas que valoran el papel educativo. Los otros hogares, muchas veces, no colaboran y hasta obstaculizan la labor escolar, y provocan, en algunos casos, situaciones incómodas entre la escuela y la casa, ya que fomentan algunos problemas en las niñas y los niños, tales como: el bajo rendimiento académico, el ausentismo y la deserción, entre otros (Cascante, Mora, y Pizarro, 1996).

La buena comunicación y el acercamiento del docente y las madres y los padres de las y los estudiantes, tiene un efecto positivo en la motivación de los y las menores hacia el estudio. De acuerdo con Monereo y Solé (1996, p.133) “[...] es necesario que el alumno se dé cuenta y perciba esa colaboración y comunicación de una manera concreta y continuada a partir de entrevistas y reuniones entre profesores y la familia".

Es innegable e ineludible la necesidad de que la familia asuma un papel protagónico, de apoyo, de solidaridad, con una actitud cooperativa hacia la institución educativa, para que, de manera conjunta, logren la permanencia de los educandos en la escuela y alcancen sus objetivos de favorecer la formación de personas saludables en su amplio sentido. 


\section{PAPEL DE LA ORIENTACIÓN EDUCATIVA}

No todos los grupos familiares tienen las condiciones más favorables, ni las características personales ideales para proveer a sus miembros menores de oportunidades de desarrollo que les faciliten ser personas de bien y autorrealizadas. En algunas ocasiones, la baja escolaridad de los padres y las madres puede limitar las opciones que estimulan el desarrollo integral de los niños y las niñas. Sin embargo, la posibilidad de que las y los responsables de éstos, se conozcan mejor, desarrollen sus potencialidades, brinden cariño, comprendan con una mayor tolerancia y aprendan estrategias que les apoyen en el adecuado desempeño de su papel, no está limitado, exclusivamente, a su nivel educativo, pues existe la posibilidad de aprender y de modificar los hábitos y las actitudes, sin importar las circunstancias.

Ante ese reto, el profesional en Orientación asume una gran responsabilidad social, en particular cuando está ubicado en el ámbito educativo, porque no puede obviar los cambios y las problemáticas de la sociedad que afectan, de manera directa a la familia. Para Villarreal (1997), los Departamentos de Orientación de las escuelas, entre otras responsabilidades, deben atender a los y las estudiantes en relación con la formación personal, las relaciones humanas, el sentido de vida, y el éxito escolar. Justamente, para poder llevar a cabo estas tareas se debe considerar la realidad en la que está sumida la población y la situación de las familias que la conforman.

El orientador no cuenta, en muchos casos, con el tiempo y los recursos materiales requeridos para generar y ejecutar sus procesos educativos, a causa de que la gran cantidad de hogares y estudiantes por atender, además de un sinnúmero de labores inherentes a su cargo; sin embargo, posee la formación psicosocial, pedagógica y técnica para liderar acciones concretas dirigidas a la familia y generadas desde su campo o, bien, conjuntamente en equipos de trabajo, conformados con otros profesionales afines.

En Costa Rica, el Consejo Nacional de Rectores (CONARE) y el Ministerio de Educación Pública (MEP) (1992) se ubicaron en la realidad del momento y realizaron proyecciones con miras al siglo XXI y sugirieron experimentar nuevos modelos de orientación que involucraran la participación activa de las madres y los padres de familia y otros actores de la comunidad, en los que se pudiera ver al orientador como un profesional que dinamizara procesos y no sólo como su ejecutor.

Los cambios que se generan, constantemente, en la sociedad obligan a que la labor orientadora, sus enfoques y sus estrategias se modifiquen para poder lograr las metas propuestas. En este sentido, Salas (1992), considerando las posibilidades limitadas que el orientador tiene para estar en contacto directo con sus orientados en los centros educativos, expone la necesidad de buscar un apoyo concreto de los profesores, los maestros y otros compañeros de la institución educativa que muestren compromiso, preparación y disponibilidad para capacitarse. Según esta autora, en esto radica el éxito de la orientación en las escuelas, ya que se implementa como "[...] una responsabilidad cooperativa de la comunidad escolar, lo que permite diversificar las formas de apoyo al proceso de orientación del individuo" (p. 83).

Es así como, en aras de establecer acciones más específicas, dirigidas a la familia en conjunto con otros profesionales, las premisas de la orientación familiar planteadas por Villarreal (1992 y 2001) descritas a continuación, pueden cimentar planes y proyectos que el orientador ha de promover y dirigir, desde los diferentes niveles en los que se puedan implementar.

El enfoque de la orientación familiar debe ser educativo, pues se parte de la idea de que en la familia se aprende, por tanto, ésta tiene capacidad de transformar a sus miembros, y la Orientación debe estimular ese potencial. Primordialmente, se pretende brindar formación en aquellos aspectos 
que favorecen la convivencia, propiciando la práctica de estilos de comunicación más saludables, desarrollando la inteligencia emocional y motivando el contacto con otros seres humanos.

Entre los propósitos de esta área de acción, se destacan: el mejorar la calidad de vida de las personas que conforman la familia, y el lograr que las personas adultas refuercen en los niños la autoconfianza, el autorrespeto y la convicción de ser amado.

También se pretende ofrecer asesoría a las familias, en relación con su dinámica, organización e identificación de estrategias, para favorecer "[...] cambios significativos que permitan a las familias cumplir con sus tareas socio-educativas" (Villarreal, 1992, p.52).

Para llevar a la práctica los principios mencionados, la autora recomienda que las estrategias por utilizar deben dar la oportunidad a los individuos de participar en los diferentes procesos, de experimentar y de trazar cambios a partir de su realidad.

Desde las instituciones educativas se pueden formar pequeños grupos de docentes, de estudiantes, de madres o padres, y suscitar temáticas de trabajo que favorezcan la vida familiar saludable, tales como el desarrollo de habilidades para vivir en ella, la relación de pareja y la preparación para el matrimonio, entre otras. Uno de los objetivos de la orientación personal-grupal es la exploración de ideas, actitudes, sentimientos y conductas que faciliten el desarrollo tanto de la persona como del grupo (Sanz, 2001). Con base en este fundamento teórico, la modalidad aplicada a la realización de proyectos de atención a la familia y a otros grupos, mediante un proceso de asesoramiento, permite la posibilidad de desarrollar algunas competencias en las y los participantes tales como: aprender a escuchar, mostrar empatía, pensar críticamente, expresar sentimientos, tomar decisiones apropiadamente, solucionar problemas, aumentar el conocimiento de sí mismo, asumir tareas con responsabilidad y propiciar la autogestión.

Otras ventajas del trabajo con grupos son:

- $\quad$ Los y las facilitadoras utilizan eficiente y efectivamente el tiempo.

- Los y las participantes aprenden unos de otros.

- Los miembros del grupo pueden ofrecer un mayor apoyo y estímulo entre ellos y ellas.

- $\quad$ El grupo puede ofrecer espacios de observación y de exploración de alternativas novedosas a los y las facilitadoras.

- Las personas tímidas reducen la tensión.

- $\quad$ El compañerismo y el sentido de pertenencia promueven un ambiente cálido y seguro, que favorece el aprendizaje.

Con esta manera de trabajar, el profesional de orientación cumple un papel determinante en la consecución de los objetivos y las metas planteadas, por lo que ha de desarrollar y poner en práctica una serie de destrezas y actitudes pertinentes a su función, con el fin de facilitar el cambio y liderar, adecuadamente, el proceso.

Para el progreso de la conducción del grupo es primordial que el facilitador o la facilitadora promueva la interacción entre los miembros del grupo, y estimule la participación activa, así como el apoyo a las y los participantes en la definición de los objetivos y las metas. Además, ha de atender necesidades individuales que se generen en el grupo, sin dejar de vigilar y afrontar las situaciones conflictivas que puedan presentarse (Sanz, 2001). 


\section{GRUPO DE ORIENTACIÓN}

Esta modalidad fue utilizada como una experiencia de capacitación para personas que estaban interesadas en el trabajo con padres y madres de familia. A continuación, se informa sobre la actividad.

\section{ASPECTOS METODOLÓGICOS}

La experiencia que se expone, se realizó dentro del marco del proyecto de extensión: Fortalecimiento de la familia, perteneciente a la División de Educación para el Trabajo (DET) del Centro de Investigación y Docencia en Educación (CIDE) de la Universidad Nacional de Costa Rica (UNA).

Los antecedentes de esta experiencia se originan cuando, a partir del año 2002, se imparten talleres dirigidos a 16 grupos de madres y padres, realizados tanto en la Sede Central de la UNA como en diferentes instituciones educativas de la región de Heredia y San José. Durante estos talleres se fueron identificando los temas que padres y madres, consideraban de mayor necesidad, para brindarles apoyo en la labor formadora de sus hijos e hijas.

Después de llevar a cabo varias experiencias con "Escuelas para padres y madres", surgió la iniciativa de unir esfuerzos y trabajar, de forma conjunta, con el Proyecto Actualización académica para administradores educativos de la DET, con el fin de apoyar a las instituciones adscritas a éste, en el cumplimiento de su plan institucional, dentro del cual se incluía el trabajo con padres, madres o responsables de niños y niñas.

Por esta razón, se diseñó y ejecutó un Seminario-taller dirigido a funcionarias y funcionarios de esos centros educativos, con el propósito de que, posteriormente, asumieran el compromiso de planear y desarrollar "Escuelas para padres y madres" en sus lugares de trabajo. Con esta capacitación se benefició a una mayor población, pues los y las participantes serían multiplicadores del proceso.

\section{Objetivos generales}

- $\quad$ Proveer un espacio de interacción y de reflexión en torno al trabajo con madres y padres, mediante el desarrollo de talleres.,

- $\quad$ Proporcionar a las y los participantes propuestas y orientaciones específicas sobre la temática de cada taller.

- Asesorar a las facilitadoras y a los facilitadores en el proceso de planeamiento y ejecución de los talleres dirigidos a madres y padres en sus respectivos centros educativos.

\section{Objetivos específicos}

- $\quad$ Revisar aspectos necesarios para una adecuada organización y conducción de un taller para madres y padres.

- Compartir y analizar las auto-percepciones sobre la experiencia de ser padre o madre de hijos e hijas escolares para valorar y mejorar su papel.

- Tomar conciencia de las emociones y reacciones que se generan con el enojo, y plantearse alternativas para su apropiado manejo. 
- Promover la exploración y práctica de alternativas en la comunicación para mejorar y fortalecer las relaciones interpersonales dentro del núcleo familiar.

- $\quad$ Fomentar principios que susciten la práctica de ideas para fortalecer la vida en familia.

\section{Ejes temáticos}

- $\quad$ Características de un facilitador o una facilitadora.

- $\quad$ Experiencia de ser padre o madre.

- Cómo nos comunicamos en familia.

- Manejo del enojo.

- Comunicación afectiva.

- $\quad$ Mi familia: aspectos que nos fortalecen.

La metodología fue esencialmente participativa, a partir de las propias experiencias de los y las asistentes; muchas de ellos y ellas, también, padres y madres, quienes indagaron sobre los temas que se trabajaron en los talleres mediante diferentes técnicas como lecturas, conversaciones con otras personas, tareas y observaciones de la propia dinámica familiar, presentaciones en multimedia, grabaciones, entre otros.

Como parte del entrenamiento realizado, se brindó a todas las personas que participaron material didáctico con planeamientos, actividades, reflexiones y otros documentos, tales como los instrumentos de evaluación y guía para sistematizar cada una de las sesiones realizadas.

El seminario-taller se llevó a cabo en 12 sesiones; las y los participantes asistían a la Universidad cada dos semanas y se basó en un módulo que se denominó "Mi familia: fortaleciendo espacios de afecto y comunicación”. El proceso se dividió en tres etapas:

1. Presentación del proyecto a escuelas interesadas. (1 sesión)

2. Vivencia del proceso de capacitación de facilitadores y facilitadoras. (6 sesiones)

3. Implementación de los talleres en cada institución y evaluación. (5 sesiones)

\section{Acerca de las y los participantes}

Se inscribieron 44 personas, de las cuales 35 se mantuvieron a lo largo del proceso, desde su inicio en junio y hasta su culminación en noviembre.

Las y los participantes, fueron funcionarios y funcionarias provenientes de 9 escuelas denominadas de "atención prioritaria", las cuales se caracterizan por atender poblaciones con limitaciones económicas y con problemáticas sociales serias. Los y las profesionales que se entrenaron tenían su formación en las áreas de Docencia, Orientación, Sicología, Trabajo Social y Sociología.

Con la ejecución del Seminario-taller dirigido a facilitadores y facilitadoras de Escuelas para padres y madres y la reproducción de experiencias a cargo del grupo participante se generaron los resultados que a continuación se exponen. 


\section{Resultados y discusión}

\section{Fase. Realización del seminario-taller para capacitar a facilitadores y facilitadoras}

Durante la ejecución del Seminario-taller, las y los participantes no sólo se consideraron a sí mismos como futuros facilitadores, sino, también, como miembros de una familia, lo que permitió un mayor beneficio para ellas y ellos en sus labores educativas, así como también en su rol ejercido en su familia: padres, madres, hermanos e hijos u otros. Esta característica permitió una participación más vivencial en las sesiones del taller, lo que generó una revisión personal que facilitó ajustes en cada una de personas involucradas en el proceso, antes de llevar a cabo el trabajo en sus respectivas instituciones. Algunas de las fortalezas relacionadas con esta modalidad fueron: la generación de un espacio para aprender, el compartir experiencias y el expresar sentimientos y opiniones. Esto concuerda con lo dicho por Sanz (2001) en relación con los objetivos de la orientación personal-grupal, en el que destaca la exploración de ideas, actitudes, sentimientos y conductas que favorecen el desarrollo no sólo de las y los participantes, sino, también, del grupo. Al respecto, una de las participantes expresó luego de su experiencia: "[...] lo enriquecedor que ha sido escuchar las experiencias de otros centros educativos, porque siempre tendemos a consumirnos mucho en nuestro propio mundo" 4 .

Se ofrecieron técnicas que, en general, fueron identificadas como útiles, participativas y sencillas, para el trabajo con madres y padres. Alguna de las personas mencionó: "[...] una de las fortalezas que. nos han enseñado son los nuevos y diferentes enfoques con respecto a los temas. Otro punto de vista por parte de ustedes y de los compañeros que también aportaron [...] las considero nuevas alternativas o herramientas que nos van a servir para los talleres con los padres" y otra: "[...] vemos que son cosas sencillitas, el amor, el tratarse bien, la buena comunicación... las podemos hacer y eso va a solucionar muchos conflictos que tenemos... en los hogares de los niños con quienes trabajamos...".

Las opiniones plasmadas por algunos y algunas participantes, coinciden con lo expresado por Villarreal (1992), quien expone que las estrategias empleadas en el trabajo con madres y padres, deben permitir la experimentación de procesos, que favorezcan cambios y, a su vez, apoyen la realización de tareas propias de las familias.

Además, según lo mencionaron las y los participantes, en un nivel personal y familiar, identifican logros que se resumen en:

- $\quad$ La toma de conciencia de hábitos cotidianos.

- La importancia de mejorar la comunicación y de aumentar el conocimiento de la propia familia.

- $\quad$ El cambio de percepciones y de sentimientos de esperanza, entusiasmo y anhelo por mejorar las relaciones con los padres y las madres desde la escuela.

En general, las valoraciones respecto a la vivencia inicial del módulo y su potencial multiplicador, son recapituladas en las siguientes expresiones: "Creemos en el hecho de que haber vivenciado el taller, logró que entendiéramos mejor cada una de las temáticas desarrolladas y el sentir de nuestros padres y madres de familia" y "[...] realmente, muchas gracias, porque ustedes mejoran nuestras familias y nosotros tenemos la oportunidad de mejorar otras más...”.

\footnotetext{
Las expresiones de los y las participantes fueron anónimas.
} 
II Fase. Resultados obtenidos por los facilitadores y facilitadoras que implementaron "Escuelas para padres y madres" en sus respectivas escuelas

Durante la II Fase, se mantuvo la participación de nueve escuelas y se ejecutó un total de 10 proyectos dirigidos a responsables de las familias. En una de las instituciones, los facilitadores, por diferencias de disponibilidad de horarios, se dividieron en dos grupos de trabajo con madres y padres.

Entre los diferentes centros educativos se logró trabajar con 137 participantes (en su mayoría madres), lo que significa que se multiplicó el módulo facilitado por el Proyecto de la Universidad con las adaptaciones pertinentes a cada una de sus realidades de las instituciones educativas. En nueve de los proyectos realizados, se ejecutaron cinco sesiones y en el otro, un total de siete, se aduce que el tiempo y el interés por las temáticas demandaron un esfuerzo extra, por parte de los facilitadores.

Estos resultados sobrepasan la capacidad de atención, que como profesionales de orientación de la UNA, se tenían en años anteriores, por lo que se considera un logro significativo para el proyecto. Específicamente, las funcionarias y los funcionarios a cargo comunican logros significativos al concluir su experiencia, basados en la participación de las y los asistentes y en las evaluaciones orales y escritas que se dieron en los diferentes talleres.

Con el fin de organizar la información en este apartado, los resultados se organizan de acuerdo con los diferentes aspectos que se presentan a continuación.

\section{Metodología y técnicas}

En general, hubo aceptación de la metodología participativa, los espacios generados son valorados como propicios para compartir ideas y sentimientos, sin temor a la crítica. Entre las opiniones expresadas por las y los participantes se comparte la siguiente: "Es un espacio para poner en práctica la capacidad de autoanalizarse y desmitificar prejuicios".

Por otra parte, los temas presentados fueron de interés para las y los responsables de familias. Al finalizar la actividad, en algunas de las escuelas se entregaron certificados de participación a las madres y a los padres.

\section{Desarrollo personal y grupal}

Los y las asistentes se mostraron motivados al participar activamente y, en varios casos, mantuvieron el compromiso de llegar puntualmente a las sesiones. Los padres y las madres participantes mostraron deseos de aprender. En sus palabras, ellas y ellos expresaron que los talleres les han ayudado "[...] a ser mejores madres, a tener más tolerancia, paciencia, a saber cómo poner límites a sus hijos e hijas, [...] también cómo poder ser más expresivas con ellos y ellas".

En el grupo se logró, en opinión de una de las participantes, “[...] crear un ambiente de confianza y de respeto" pues conforme se llevaba a cabo el proceso había más confianza entre los miembros del grupo. La dinámica del curso trascendió, en ocasiones, de la modalidad de taller a grupo de apoyo, en la que "[...] la participación de las facilitadoras disminuyó para dar paso a una intervención grupal más activa”, como lo señaló una de las asistentes. 
Sanz (2001) menciona al respecto que en estos grupos, las personas se sienten más apoyadas, con más confianza de expresar cuando perciben que los intereses y las preocupaciones de los demás son comunes a ellos. Esto permite que los miembros del grupo trabajen juntos hacia la consecución de objetivos y metas comunes.

\section{Relación familia-institución educativa}

Se logró un mayor acercamiento a la escuela por parte de las madres y los padres. Se les involucró en forma positiva y se logró la disponibilidad hasta para participar en actividades escolares y formar grupos de apoyo académico. Y en algunos casos muy particulares, se creó un precedente, pues era la primera vez que se realizaba este tipo de actividades.

\section{Relación escuela-niños y niñas}

En dos instituciones, las y los organizadores del proyecto implementaron el cuidado de niños y niñas (guardería), mientras las madres y los padres participaban de los talleres. Se considera que esta oportunidad generó la motivación de los niños y de las niñas por las actividades escolares. Esto concuerda con la opinión de Monereo y Solé (1996), quienes plantean que el acercamiento del docente con los padres y las madres, de manera concreta y continuada, tiene un efecto positivo en la motivación del niño y la niña hacia el estudio.

\section{Logros personales y familiares de las madres y los padres}

En general, se mencionó que las y los participantes disfrutaron de la experiencia, porque les enriqueció de manera personal, lo que, también, beneficia a sus familias; algunas personas solicitaron más sesiones de trabajo y manifestaron interés por participar el año siguiente.

Algunos de los cambios implementados para mejorar las relaciones con los miembros de la familia y expresados por los mismos participantes se relacionan con:

"[...] la forma de educar y ayudar a estudiar a los hijos [...]”; "[...] cambiar la mentalidad, sobre todo, al abordar temas como el enojo [...]" y "[...] su forma de trato para con sus hijos [...]".

\section{Relación escuela-comunidad}

Se logró una mayor proyección de la institución hacia la comunidad. Entre las y los asistentes a los talleres de una de las escuelas, se encontraron personas de otras instituciones educativas y de la clínica de salud de la comunidad. Asimismo, se generó un espacio de integración entre la comunidad y el centro educativo; y se puso en evidencia la información que vislumbra algunas problemáticas que viven las familias del lugar. Entre otros datos, se conoce que algunos responsables de familia se ausentan o no se presentan a la actividad del todo, debido al tipo de labores que realizan para subsistir y, en otros, la timidez que podría estar originada en la poca o ninguna formación académica. Esto cambió la percepción inicial que se tenía sobre el desinterés por este tipo de actividades. 


\section{Impacto social}

Con la ejecución del proyecto se promovieron actitudes, valores y tradiciones de las familias que repercuten, favorablemente, en las comunidades, y que, también, robustecen a los grupos familiares que se ven afectados por los cambios, los avances y los problemas sociales. Sobre la experiencia, algunos facilitadores expresaron: “[...] logra mejorar la salud emocional de los miembros de la familia y, por tanto, de la sociedad". Fue, además, una oportunidad para levantar un listado de preocupaciones e inquietudes de las madres y los padres que serán tomados en cuenta en futuros talleres, con los fines ya propuestos en el documento.

\section{Retos a los que se enfrentaron facilitadoras y facilitadores}

Durante el transcurso de la capacitación efectuada, los miembros facilitadores del Proyecto Fortaleciendo la familia lograron identificar las mayores dificultades evidenciadas, con el fin de valorar e implementar mejoras en futuros entrenamientos.

De acuerdo con un instrumento de autoevaluación aplicado a las y los participantes se estableció que los mayores retos encontrados se centraron en: a) el motivar y mantener la asistencia de las madres y los padres, b) la disposición de tiempo extra para ejecutar talleres y llevar registros escritos, c) la escasez de dinero y recursos materiales, d) las limitaciones personales, tales como: tener que hablar de hijos sin tenerlos, superar la timidez, tolerar las diferentes formas de pensar, e) el trabajo en equipo, f) la obtención de apoyo y de compromiso de la institución, g) la ampliación de los conocimientos y h) el uso de metodologías innovadoras.

Al finalizar el seminario- taller, el Proyecto Fortaleciendo la familia, con el respaldo de Universidad Nacional de Costa Rica, se entregó un certificado de aprovechamiento al grupo de futuros facilitadores, con un total de 44 horas. Se acreditó a aquellos que cumplieron con el $80 \%$ de la asistencia a las sesiones y con la entrega de un informe final sobre el trabajo realizado en las instituciones.

\section{CONCLUSIONES}

Algunas conclusiones generadas de la experiencia realizada son:

- $\quad$ La oportunidad que tuvieron las y los participantes de vivenciar el seminario-taller no sólo enfocado a ellas y ellos como futuros facilitadores de una escuela de padres y madres, sino como miembros integrantes de una familia, les permitió tener un espacio de reflexión sobre la dinámica de su familia.

- Al efectuar las reflexiones y realizar las tareas con sus familias, promovió la concienciación de aspectos familiares que, en la cotidianidad, no se toman en cuenta, como por ejemplo evaluar cuál es su reacción en una situación que les provoca enojo, o la opinión que sus hijos e hijas tienen de ellos como madres y padres.

- Escuchar los puntos de vista de compañeros y compañeras madres y padres, sobre aspectos familiares comunes, les dio la oportunidad de ampliar su visión con respecto a temas de la familia, lo cual fue muy valioso cuando trabajaron con los grupos en sus instituciones. 
- El hecho de que las y los participantes fueran de diferentes instituciones y se dieran realimentaciones frecuentes del trabajo de campo en cada institución, permitió enriquecer la experiencia y brindó a las y los participantes una motivación y nuevas ideas, para que pudieran llevar a sus centros de trabajo.

- $\quad$ La sistematización de las técnicas que se trabajaron con las facilitadoras y los facilitadores, y todo el material brindado por el Proyecto Fortaleciendo la familia, fue de gran provecho para coordinar, planear y realizar las Escuelas de padres y madres en cada institución, de forma más organizada y efectiva.

- La motivación y el interés de las y los participantes por realizar las actividades en los diferentes talleres, así como las tareas asignadas para ser aplicadas en sus familias, permitió que el grupo fuese muy compacto y que la mayoría de quienes empezaron el seminario-taller lo culminaran.

- De acuerdo con la opinión de los participantes, la agilidad, la responsabilidad, la certeza y la organización del equipo de trabajo del Proyecto Fortalecimiento la familia significó un modelo importante para la aplicación del taller en sus instituciones.

- La respuesta positiva que generaron, tanto las facilitadoras y los facilitadores capacitadores, como las madres y los padres capacitantes en las diferentes instituciones en donde se ofrecieron los talleres, corrobora la gran necesidad que poseen los progenitores y progenitoras, responsables de familias, de espacios de reflexión y asesoramiento, que les ayuden en la delicada labor formativa de sus hijos e hijas.

\section{REFERENCIAS BIBLIOGRÁFICAS}

Cascante, I. (1992). Breve reseña de la carrera de Orientación. Escuela de Orientación Educativa. Universidad Nacional. II Jornada de Orientación Vocacional Profesional. Heredia, Costa Rica: Universidad Nacional.

Cascante, V., Mora, J. \& Pizarro, A. (1996). Estrategias que relacionan a los padres de familia con el proceso de Enseñanza y Aprendizaje en el C.T.P.A. Carrillo Circuito 05 Región Educativa Santa Cruz. Tesis. Universidad de Costa Rica.

Castells, P. (1997). La familia ¿Está en crisis? Barcelona, España: Plaza \& Janés Editores.

Consejo Nacional de Rectores (CONARE) y Ministerio de Educación Pública (MEP). (1992). La Orientación del siglo XXI. II Jornada de Orientación Vocacional Profesional. Heredia: Oficina de Publicaciones Universidad Nacional

Monereo, C. \& Solé, I. (1996). El asesoramiento psicopedagógico: una perspectiva profesional y constructivista. Madrid, España: Alianza.

Pereira, M. T. (2004). Orientación educativa. San José, Costa Rica: EUNED. 
Proyecto Estado de la Nación en Desarrollo Humano Sostenible. (2006). Resumen del Duodécimo Informe Estado de la Nación en Desarrollo Humano Sostenible. San José, Costa Rica: Autor.

Rockwell, R. (1995). Parents and Teachers as partners. Texas, United States: Fort Worth.

Salas, E. (1992). ¿Cómo orientar? Santiago, Chile: Universitaria.

Sanz, O. (2001). Orientación psicopedagógica y calidad educativa. Madrid, España: Pirámide Ediciones.

Villarreal, C. (1992). La orientación familiar: una opción educativa. Revista Educación, Vol.16, N 2, 51-63.

Villarreal, C. (Compil.). (1997). Memoria del Seminario La orientación familiar: hacia una nueva perspectiva de vida en familia, realizado en 1995 en la Facultad de Educación de la UCR (iniciativa de la Escuela de Orientación y Educación Especial de la UCR, como un programa de acción social y en coordinación con el Departamento de Orientación y Vida Estudiantil del MEP). San José, Costa Rica: Universidad de Costa Rica.

Villarreal, C. (2001). La humanización de la familia: una responsabilidad educativa. Revista Educación, Vol. 25, №1, 137-150.

Zamudio, L. (febrero/marzo, 2008). Un trabajo por la familia, en el contexto municipal. Red de Gestores Sociales, 36. Recuperado el 3 de abril, 2009, de www.rgs.gov.co/img upload/ca2aff77eed2a9b504622b634052d133/RGS38 070508color FINAL.pdf 